Jan Niehues, Peter Lehmann and Weichang Xie

Low coherent Linnik interferometer optimized for use in Nano Measuring Machines

This publication was developed as part of the event:

$56^{\text {th }}$ IWK, International Scientific Colloquium, IImenau University of Technology, 12 - 16 September 2011 / Faculty of Mechanical Engineering, IImenau University of Technology

Ilmenau University of Technology IImenau, 2013

URN: urn:nbn:de:gbv:ilm1-2013200109 


\title{
LOW COHERENT LINNIK INTERFEROMETER OPTIMIZED FOR USE IN NANO MEASURING MACHINES
}

\author{
Jan Niehues, Peter Lehmann, and Weichang Xie \\ University of Kassel, Department of Electrical Engineering/Computer Science, Chair in \\ Measurement Technology
}

\begin{abstract}
The precise acquisition of three-dimensional geometrical data in micro- and nanotechnology is crucial for advanced fabrication processes. Scanning white-light interferometry (SWLI) achieves nanometer resolution in the axial direction, but the lateral resolution is far more critical. The requirements for an areal measuring optical sensor in a Nano Measuring Machine are very high. The resolution in every dimension and the working distance have to be as high as possible. In contrast to a Mirau interferometer a Linnik interferometer does not need any components in front of the objective lens. This benefit permits both, a long working distance and a high lateral resolution.

In the EC-funded project "NanoCMM" we developed a Linnik interferometer providing a working distance of more than $5 \mathrm{~mm}$ and a lateral resolution of $0.3 \mu \mathrm{m}$. With this interferometer we measured the full modulation depth and the rectangular shape of a pitch standard close to the Rayleigh resolution limit.
\end{abstract}

Index Terms - white-light interferometry, Linnik interferometer, lateral resolution, nano measuring machine, UV illumination

\section{INTRODUCTION}

Production processes in micro- and nano-technology require adequate measuring instruments to obtain three-dimensional geometries. Due to increasing production requirements, the lateral resolution plays a more and more crucial role. SWLI is a fast and highly accurate method to measure 3D topographies of technical objects. ${ }^{1}$ It is particularly well suited for the measurement of step height structures e.g. in microelectro-mechanical systems (MEMS). The measuring principle is based on a so-called depth scan. During this scan the optical path-length difference between the measuring and the reference arm of the interferometer is varied in small steps. At every scanner position an interferogram is recorded by a digital video camera. ${ }^{2}$ This results in a separate interferogram for each camera pixel known as correlogram. Shadowing effects are mostly avoided because the optical axes of the illumination and the measurement path of the interferometer are the same. ${ }^{2,3}$

To obtain the 3D-topography from the measured interference patterns different signal processing methods are used. In a first step the maximum position of a correlogram's envelope is usually determined. This method can be realized by contrast or by spatial frequency domain analysis. ${ }^{5}$ By additionally evaluating the phase of the interference signal sub-nanometer height resolution can be achieved. ${ }^{1,5,6}$ To solve the ambiguity problem of phase evaluation the envelope is used to determine the corresponding fringe order. This procedure results in a comparable accuracy to conventional phase-shifting interferometry. Due to the fringe order determination also height steps can be measured correctly. ${ }^{6,8}$

The subject we address in this paper is the lateral resolution in SWLI. For many measuring objects the height resolution of SWLI is more than sufficient, but the increasing miniaturization also demands better lateral resolution. There are different approaches to characterize the lateral resolution of microscopic 3Dmeasuring systems. ${ }^{9,10}$ However, none of these corresponds to the measuring results we obtained from a UV illuminated Linnik type SWLI.

\section{LATERAL RESOLUTION IN SWLI}

For an optical imaging system it is generally assumed that the lateral resolution corresponds to the optical resolution represented by the Rayleigh criterion: $\delta=0.61 \lambda / N A$, or the Sparrow criterion: $\delta=0.47 \lambda / N A$, where $\delta$ is the smallest distance of two separated points on a measuring object. These criterions are based on linear system theory. Since an optical profiler differs from a 2D imaging system, the different height levels of an object may lead to strong nonlinear effects. For such optical profiling systems no satisfying definition of the lateral resolution exists so far. Although there are a lot of publications about height resolution in SWLI, the lateral resolution has barely been examined. P. de Groot et al. have introduced the instrument transfer function (ITF). ${ }^{10}$ This function is an extension to the 3D situation of the well-known modulation transfer function (MTF) in 
optical imaging. For a height step of the measuring object of only $40 \mathrm{~nm}$ the resulting ITF is close to a typical MTF. Here the interference intensity is proportional to the surface height and interferometric imaging behaves linear.

Corresponding to tactile instruments the German guideline VDI/VDE 2655-1.1, which deals with the calibration of interference microscopes, introduces a short cutoff wavelength related to the surface structure. ${ }^{9}$ In this approach the measured height of a rectangular grating structure decreases if the spatial frequency increases, i.e. the instrument shows a low pass characteristic. According to the guideline the minimum resolvable pitch value in SWLI corresponding to the short cutoff wavelength has to be 2 times to 3 times the value of the lateral resolution determined by the Rayleigh criterion. If the full modulation depth of a periodic object structure has to be determined, the pitch value should be 4 times to 6 times greater than the Rayleigh resolution. According to our measuring results the interferometer behaves non-linear, if the maximum height differences of structured surfaces are not small compared to a quarter of the wavelength of light. In addition, the measurement and evaluation procedures are strongly non-linear. Small changes of the input parameters can lead to rapid changes in the output.

However, an optical profiler differs from a 2D imaging system, since two points on a height profile attributed to different height levels need to be laterally resolved. Fig. 1 illustrates the basic difference in lateral resolution between $2 \mathrm{D}$ imaging and $3 \mathrm{D}$ measurement. Fig. 1a shows a modulated amplitude grating. The two curves represent cross sections of point spread functions for the two centered bright lines. According to the Rayleigh criterion the least resolvable pitch value is $\Lambda_{2 \mathrm{D}}=\delta=0.61 \lambda \mathrm{NA}$, with the resolution limit $\delta$, the numerical aperture NA and the center wavelength $\lambda$ of the used light.

In order to measure the amplitude of a 3D height modulated object, i.e. a phase object, at least one point on each height level has to be resolved. Accordingly, the resolvable pitch value increases by a factor of two so that $\Lambda_{3 \mathrm{D}}=2 \delta=1.22 \lambda / N A$.

Another point influencing the lateral resolution is the object's aspect ratio. The experimental results shown in this paper have aspect ratios below one. The same is true for most objects in practice. Hence, the results presented in the following section apply to the majority of 3D microstructured measuring objects.
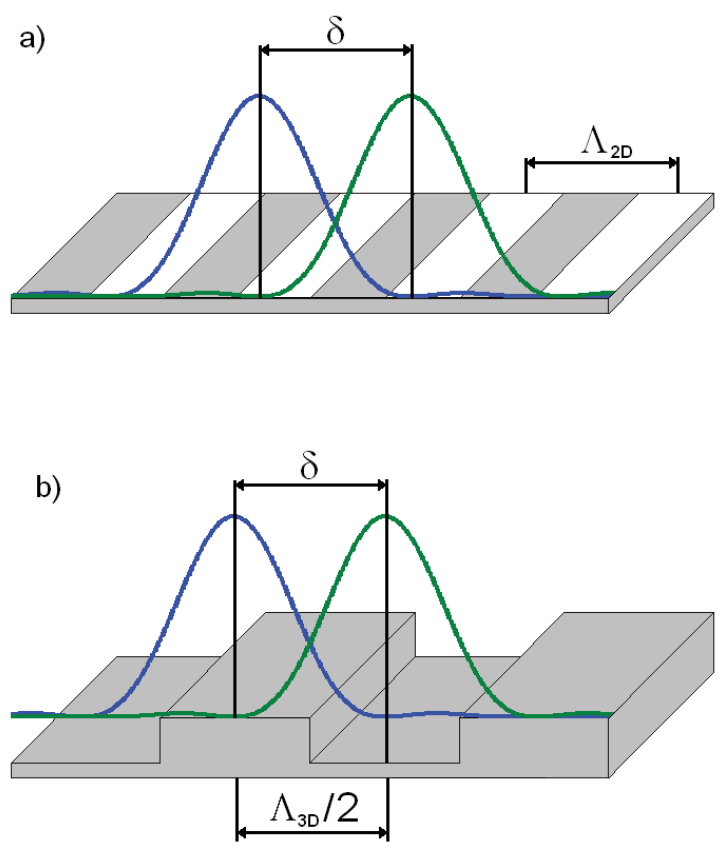

Fig. 1: a) Cross section of the point spread functions of two points on a $2 \mathrm{D}$ intensity modulated object. The distance $\delta$ is the lateral resolution corresponding to the Rayleigh criterion. $\Lambda_{2 \mathrm{D}}$ is the minimum resolvable pitch value of the grating.

b) Intensity point spread functions of two points on a $3 \mathrm{D}$ height modulated object. The distance $\delta$ remains the same. In order to determine the height of the grating, one point on every height level has to be measured. In this case the smallest resolved pitch value is $\Lambda_{3 \mathrm{D}}=2 \Lambda_{2 \mathrm{D}}$.

\section{RESOLUTION OPTIMIZED LINNIK INTERFEROMETER}

The lateral resolution of an optical microscopic instrument depends on the wavelength of the used light and the NA of the objective lens. In the project "NanoCMM" (Nano Coordinate Measuring Machine) a white-light interferometer should be integrated into a nano measuring machine. The objective lens of the interferometer should not reach into the measuring volume of the machine to prevent mechanical damage of the machine or the measuring object. Thus, a minimum working distance of $5 \mathrm{~mm}$ at best possible lateral resolution is required. These two demands counteract each other. Depending on the magnification in SWLI three types of interference objectives are used.

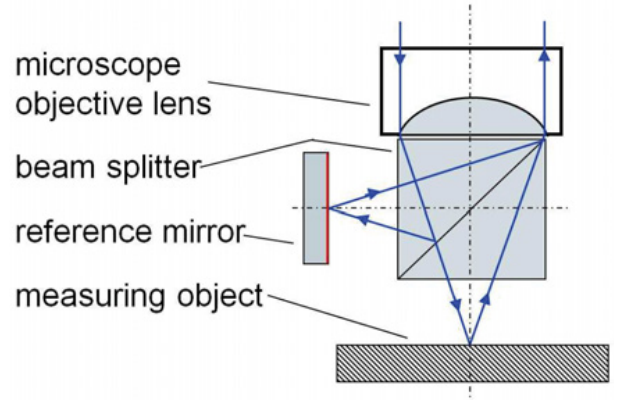

Fig. 2: Scheme of Michelson type interference objective. 
Systems with small magnifications from $1 \mathrm{x}$ to $5 \mathrm{x}$ are usually realized in the Michelson arrangement with a beam splitter under the lens as shown in Fig. 2. In this setup the reference mirror is placed outside the imaging path. At these low magnifications the aperture angle is rather small and the field of view is quite big so that a mirror inside the imaging path would block too much light coming from the object.

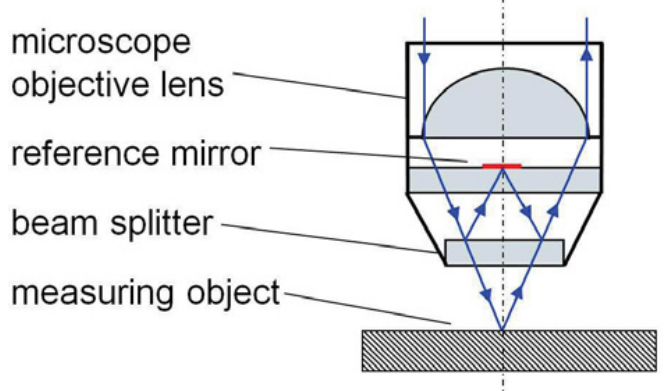

Fig. 3: Scheme of Mirau type interference objective.

Most white-light interferometers are equipped with a Mirau objective as shown in Fig. 3. Here two plane parallel glass plates are placed in front of the objective lens. This setup is space-saving and robust against mechanical influences. However, in certain cases an influence of the reference mirror on the measuring results can be observed..$^{11,12}$

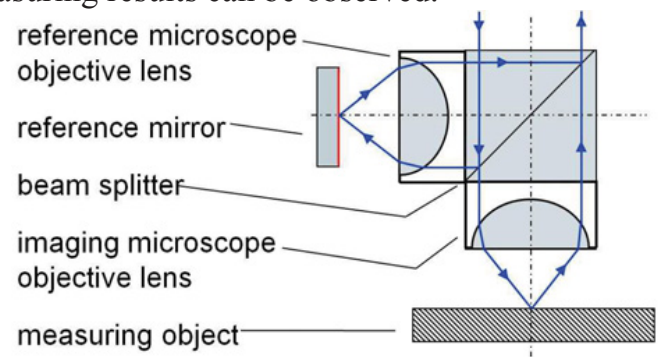

Fig. 4: Scheme of Linnik type interference objective.

Fig. 4 shows the type of objective providing the highest NA. In this Linnik type objective no components in front of the objective lens are needed. In order to achieve an equal optical path length in the reference path and to reduce aberrations a second objective lens is needed. Due to this second lens a Linnik setup is quite expensive. Another reason why it is rarely used is the demanding adjustment and its sensitivity against mechanical influences.

However, in the environment of a nano measuring machine no mechanical influences affect the interferometer. Under these conditions the Linnik setup is the best choice to optimize the lateral resolution.

\section{NANOCMM SETUP}

Fig. 5 shows the schematic setup of the complete Linnik interferometer. The illumination unit comprises two LEDs emitting at different center wavelengths. Thus, without touching the interferometer measurements using different wavelength regimes can be made. The condenser lens images the diffuser plate into the entrance pupil of the microscope objectives to obtain a homogenous illumination. The objectives corrected for the near UV range show a 50x magnification and a rather long working distance of $7 \mathrm{~mm}$. With the NA of 0.55 the Rayleigh criterion results in a lateral resolution of $0.67 \mu \mathrm{m}$ for a wavelength of $600 \mathrm{~nm}$ and $0.44 \mu \mathrm{m}$ for $395 \mathrm{~nm}$.

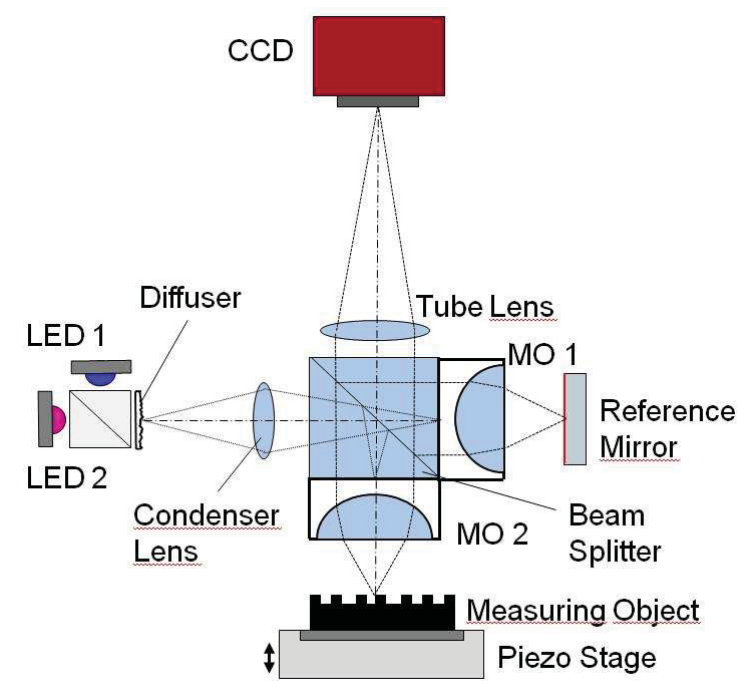

Fig. 5: Scheme of a Linnik type interferometer equipped with two LEDs emitting at different center wavelengths and two microscope objectives MO 1 and MO 2.

The tube lens also works in the near UV range. Other optical components like the diffuser and the beam splitter in front of the LEDs are made from fussed silica to work in the UV range. Since the maximum output power of available LEDs rapidly falls below $400 \mathrm{~nm}$ this currently limits the shortest usable wavelength.

The CCD comprises 1392 x 1040 pixels on a $1 / 2$ " chip resulting in a lateral sampling interval of only $100 \mathrm{~nm}$. To perform the SWLI depth scan outside the nano measuring machine the measuring object is placed on a piezo stage. The distance between the measuring object and the objective lens is varied in steps of $20 \mathrm{~nm}$ while the camera takes an image after each step. The position of the reference mirror is fixed during the measurement.

Due to the fact that the interference takes place behind the objective lenses dispersion effects have to be minimized. Thus, the beam splitter cube has a high planarity and low dimensional and angular tolerances. ${ }^{13}$ This ensures that in both arms of the interferometer the optical path lengths in glass are nearly the same.

The mechanical setup has to provide a number of adjustment axes with low tolerances as shown in Fig. 6. At first, both objective lenses have to be placed exactly onto the optical axis. Only small tilt angles 
would lead to significant dispersion effects in the measuring results.

Secondly, the object and the reference mirror have to be placed exactly in the working distance (WD) given by the focal length of the objective lens. Just as important is the axial objective lens distance (OLD), i.e. the distance between the microscope objectives and the center of the beam splitter. Due to the relatively small field of view and the high NA the system is quite tolerant against a small tilt of the reference mirror or the measuring object. All these requirements lead to the need of high accuracy mechanical adjustment capabilities.

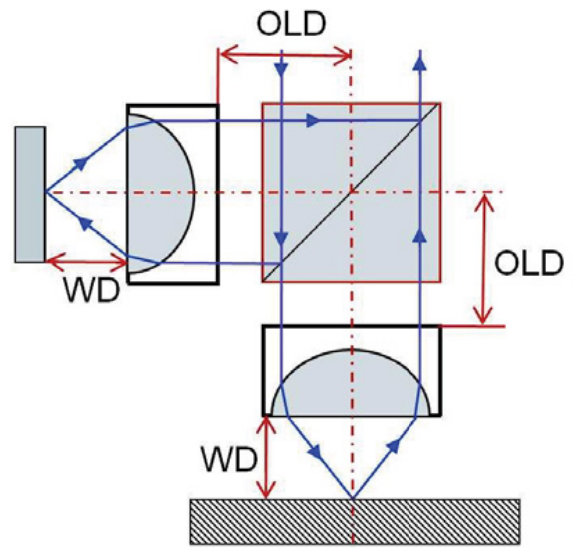

Fig. 6: Scheme of a Linnik setup with the working distance (WD), the axial objective lens distance (OLD) and a highly planar beam splitter cube with low dimensional and angular tolerances.

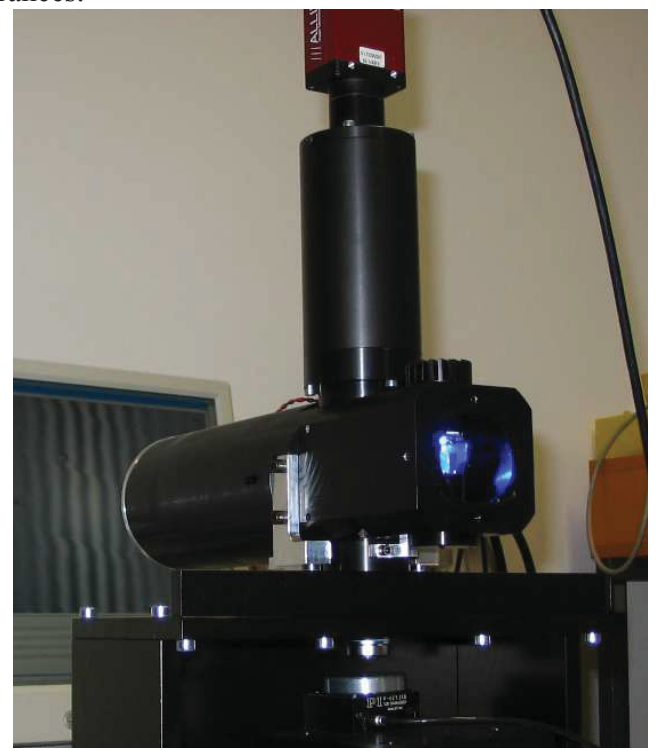

Fig. 7: Photograph of a prototype of an industrial version of the Linnik sensor.

Fig. 7 shows a photograph of the final sensor. The crucial point is to minimize the mechanical adjustment axes. Otherwise it is almost impossible to see interference fringes on the CCD. An xyzpositioning stage is needed underneath the sensor to move the object into the correct measurment position. In this setup the depth scan is performed via a pizo Zstage.
The sensor after integration into the CMM can be seen in Fig. 8. Here the proper positioning of the object and the depth scan are realized by the high presicion axes of the machine.

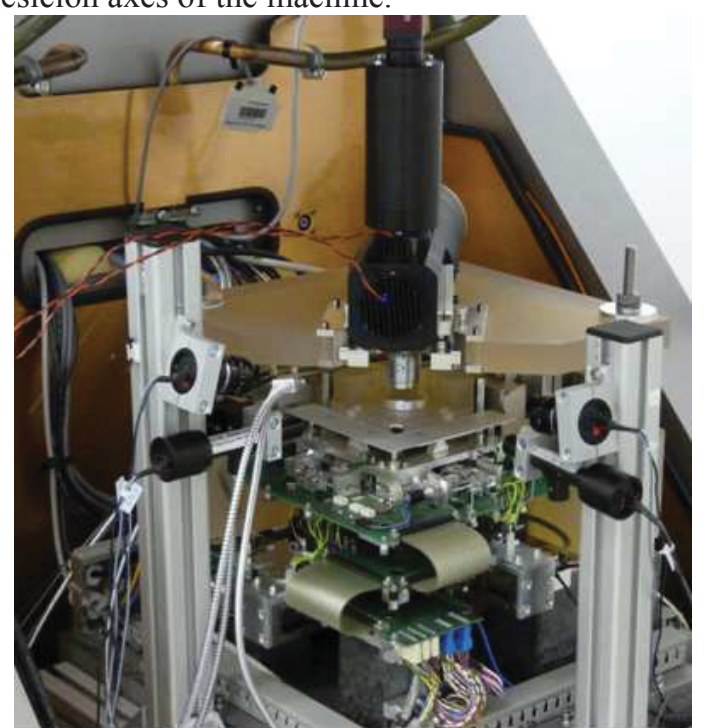

Fig. 8: Photograph of the Linnik sensor integrated into a nano measuring machine at the TU Ilmenau.

\section{EXPERIMENTAL RESULTS}

According to the mentioned VDI/VDE guideline ${ }^{8}$ a well suited lateral resolution standard shows rectangular grating structures of different pitch lengths down to the Rayleigh resolution and below. The grating amplitude should be sufficiently high, so that the measurement results obtained on the resolution standard give a realistic impression with respect to applications in micro-technology.

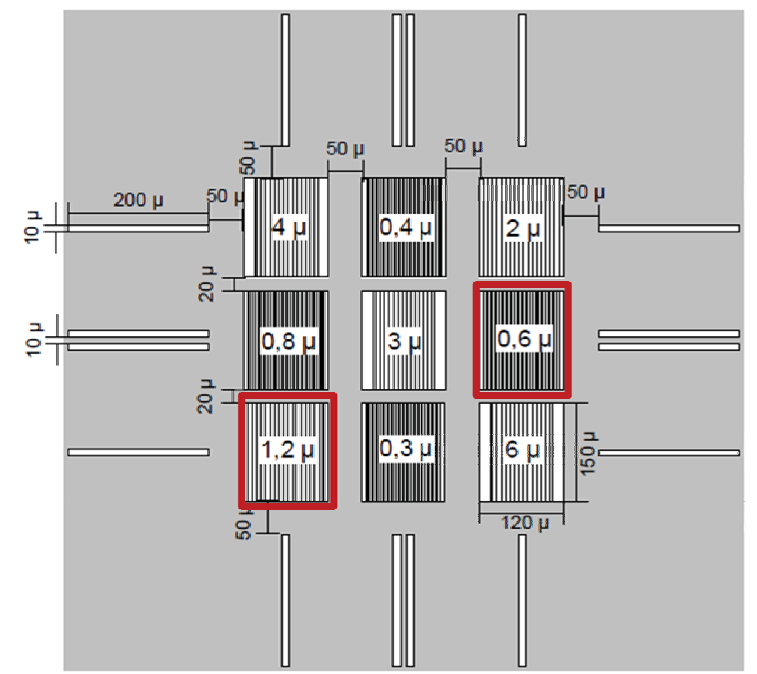

Fig. 9: Resolution standard RS-N manufactured by Simetrics comprising nine rectangular gratings with pitch lengths from $0.3 \mu \mathrm{m}$ to $6 \mu \mathrm{m}$. $^{15}$

The aspect ratio of the rectangular structures as well as the ratio between the height of the grating structure and the central light wavelength influence the occurrence of edge effects like the batwing effect. 
For the investigation documented here we choose the resolution standard RS-N made by Simetrics. The arrangement of the standard and the pitch values are shown in Fig. 9. The smallest pitch values range from 0.3 to $6 \mu \mathrm{m}$. The nominal peak-to-valley amplitude is $190 \mathrm{~nm}$. Due to the etching process the depth becomes smaller as the grooves become narrower. Considering Fig. 1 the least resolvable pitch value $\Lambda_{3 \mathrm{D}}$ in the $3 \mathrm{D}$ case is $1.33 \mu \mathrm{m}$ assuming a light wavelength of $600 \mathrm{~nm}$ and $0.88 \mu \mathrm{m}$ for $395 \mathrm{~nm}$.

In the following we show results related to the pitch values of $1.2 \mu \mathrm{m}$ and $0.6 \mu \mathrm{m}$. Illuminating with $600 \mathrm{~nm}$ the interferometer should not be able to resolve the $1.2 \mu \mathrm{m}$ nor the $0.6 \mu \mathrm{m}$ pitch length. According to the ITF as defined by de Groot et al. ${ }^{9}$ the measured normalized modulation depth should be clearly below one at a pitch length of $1.2 \mu \mathrm{m}$ and close to zero at $0.6 \mu \mathrm{m}$ pitch. Due to non-linear transfer characteristics of the interferometer the measurement results differ from the theoretical considerations.

\subsubsection{Interference Fringes and Signals}

In order to understand the complex measurement process of an optical profiler such as a white-light interferometer it is helpful to take all available information into account. The first impression of what happens at the surface under investigation gives us the CCD image of an interference pattern.

Fig. 10 shows such an image of the standard at a pitch value of $0.6 \mu \mathrm{m}$ illuminated with a white-light LED emitting at a center wavelength of $600 \mathrm{~nm}$. The rectangular surface structure and the interference fringes are hard to see at this wavelength.

Reducing the wavelength to $395 \mathrm{~nm}$ by use of a UV LED the contrast rises as Fig. 11 demonstrates. The rectangular structure is clearly visible and also interference fringes appear. This example shows that a relatively small wavelength reduction leads to a significant contrast enhancement in interference microscopy.

Figures 12 and 13 show intensity signals of a single CCD pixel recorded during the depth scan of the interferometric measurement. The signal in Fig. 12 corresponds to the resolution standard with a pitch value of $0.6 \mu \mathrm{m}$ illuminated by a white-light LED. The center wavelength of the illuminating light can be obtained from the cycle length of the interfernce fringes.

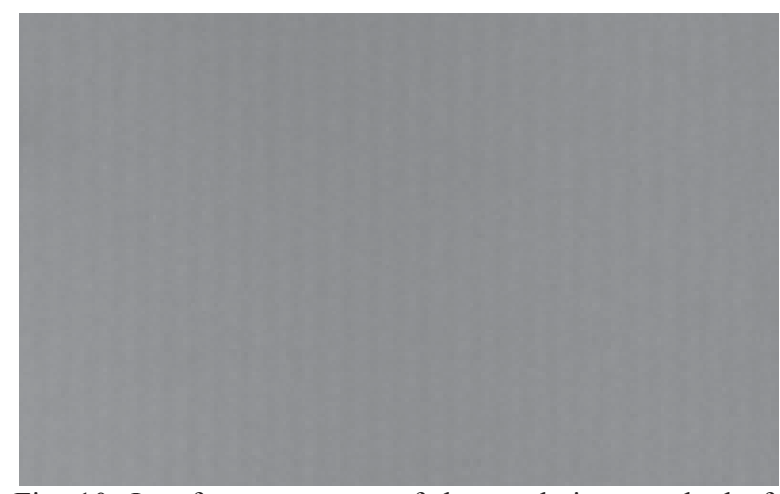

Fig. 10: Interference pattern of the resolution standard of $0.6 \mu \mathrm{m}$ pitch illuminated with a white-light LED emitting at a center wavelength of $600 \mathrm{~nm}$.

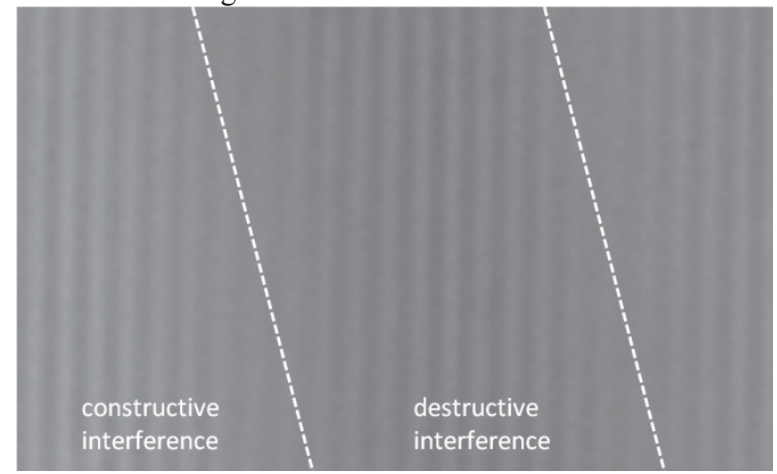

Fig. 11: Interference pattern of the same resolution standard as in Fig. 10 but with UV LED illumination (center wavelength $395 \mathrm{~nm}$ ).

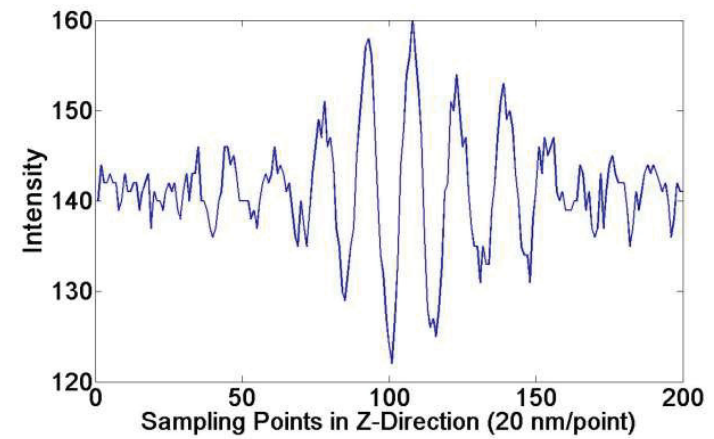

Fig. 12: Interference signal (correlogram) recorded by a single CCD pixel on a silicon standard with a pitch length of $0.6 \mu \mathrm{m}$ using white-light LED illumination $(600 \mathrm{~nm}$ center wavelength).

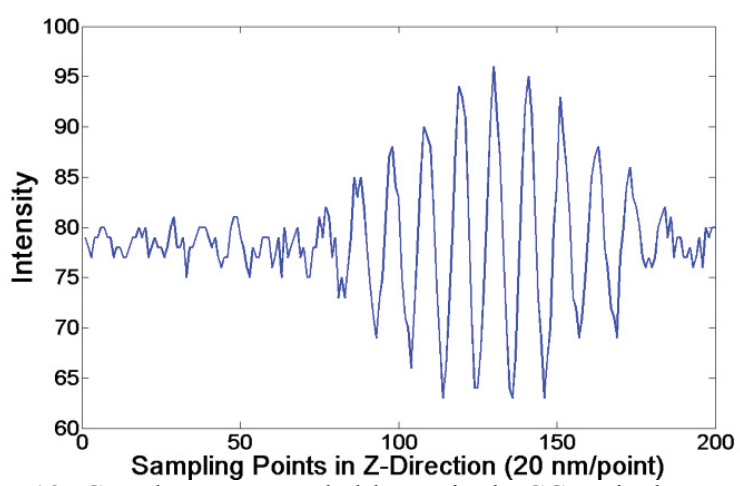

Fig. 13: Correlogram recorded by a single CCD pixel on a silicon standard with a pitch length of $0.6 \mu \mathrm{m}$ using UV LED illumination (395 $\mathrm{nm}$ center wavelength). 
Due to the insufficient lateral resolution the surface acts like a noisy mirror. The modulation depth of the signal depends on the surface strucure but also on the intensity of the illuminating light. The optical output power of the white-light LED is much higher compared to the UV LED. Thus, the interference contrast is high enough to evaluate the signals but except for some noise all signals appear at the same height level.

Fig. 13 shows a signal occuring at the same resolution standard with $0.6 \mu \mathrm{m}$ pitch but under illumination with the UV LED emitting at $395 \mathrm{~nm}$. The shorter wavelength results in a reduced cycle length of the interfernce fringes. In addition, the narrow spectrum of the UV LED leads to a longer coherence length which increases the number of cycles in the interfernce signal. Although the intensity of the UV LED is much lower the modulation depth is quite as big as in case of the white-light LED. This shows that the higher contrast in the interference pattern (Fig. 11) increases the modulation depth of the interfernce signal.

\subsubsection{Profile Measuring Results}

In order to demonstrate the resolution enhancement with respect to conventional white-light interferometers the measuring results of the Linnik interferometer are compared to those obtained by a Mirau interferometer. Both instruments provide the same 50x magnification and nearly the same NA ( 0.55 for the Linnik and 0.5 for the Mirau interferometer). A profile section of a measuring result recorded with a Mirau interferometer on the $1.2 \mu \mathrm{m}$ pitch grating illuminated with a white-light LED is shown in Fig. 14a. The curve at the top shows the result of the envelope evaluation. The measured height of the standard is twice as high as expected whereas the measured profile reproduces the rectangular shape correctly. Although the batwing effect cannot be seen very clearly because of the small cycle length of the profile we assume that this effect causes the height measuring error. ${ }^{13}$ In the result of the phase evaluation this error leads to an incorrect fringe order resulting in additional height steps of half the wavelength. In practice such results are particularly critical because the shape of the measuring object seems to be obtained correctly. If the correct amplitude is not known, there is no indication that the result is incorrect. These height steps of $\lambda / 2$ lead to a measured amplitude of about $500 \mathrm{~nm}$. The results displayed in Fig. 14b are measured by the Linnik interferometer. The result of the envelope evaluation shows increased noise compared to the result according to Fig. 14a. This is a consequence of the longer coherence length of the blue LED used in the Linnik interferometer. However, the result is sufficiently good to find the right fringe order. Hence, from the phase evaluation the correct height value of $190 \mathrm{~nm}$ can be obtained, although batwings are visible at the edges of the profile. a)
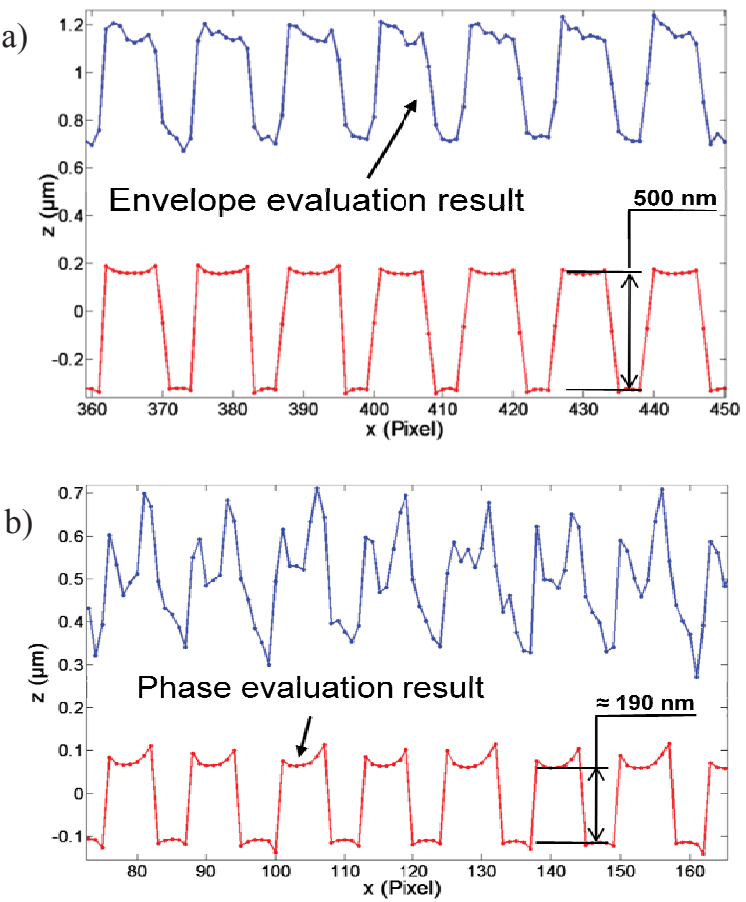

Fig. 14: Profiles measured on a silicon standard with a pitch length of $1.2 \mu \mathrm{m}$ and $190 \mathrm{~nm}$ peak-to-valley amplitude. The sampling interval given by the pixel pitch is $100 \mathrm{~nm}$, a) Profile section obtained with a 50x Mirau interferometer (center wavelength: $600 \mathrm{~nm}$ ), b) Profile section measured with a 50x Linnik interferometer (center wavelength: 450 $\mathrm{nm}$ ), the upper curve represent the height values corresponding to the envelope position, the lower curves are based on phase evaluation techniques.

The profiles plotted in Fig. 15 again show a comparison of a conventional Mirau and the resolution optimized Linnik interferometer. The pitch value of the resolution standard is now $0.6 \mu \mathrm{m}$. Fig. 15a demonstrates that the grating structure is no longer resolved by the Mirau interferometer. The minimum resolvable pitch value $\Lambda_{3 \mathrm{D}}$ for the $3 \mathrm{D}$ case (see Fig. 1) is twice the Rayleigh resolution of $0.73 \mu \mathrm{m}$. In this case the theoretical consideration that the grating structure cannot be resolved agrees with the measuring results. For the results according to Fig. $15 \mathrm{~b}$ the wavelength used in the Linnik interferometer was $395 \mathrm{~nm}$ (UV-LED). This reduced wavelength theoretically leads to a minimum resolvable pitch value of $\Lambda_{3 \mathrm{D}}=0.88 \mu \mathrm{m}$ since the Rayleigh resolution is $0.44 \mu \mathrm{m}$. However, the measuring results show the correct shape of the grating and the measured step height differs only slightly from its nominal value. These results give rise to the conclusion that, apart from the batwing effect, the Rayleigh criterion known from 2D imaging seems to work better than the lateral resolution according to the 3D case of Fig 1. The reason for this can be found in the special instrument transfer characteristics of a vertical scanning white-light interferometer as it is shown in the following section. 
a)

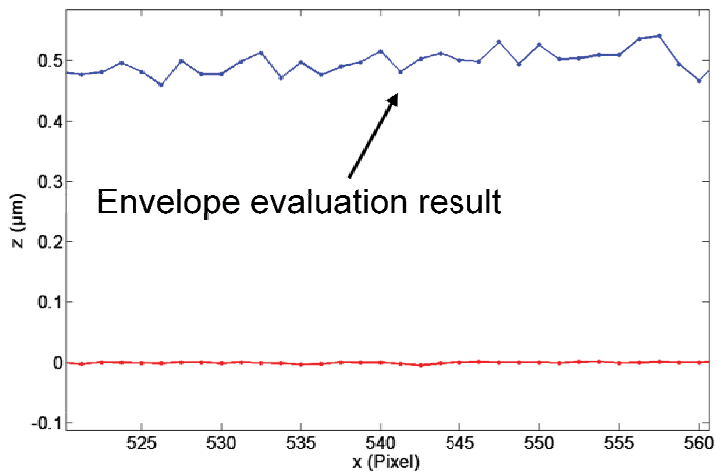

b)

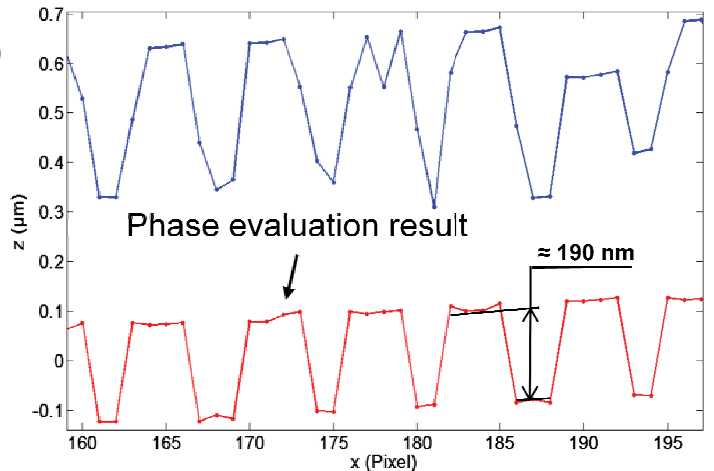

Fig. 15: Profiles measured on a silicon standard with a pitch value of $0.6 \mu \mathrm{m}$ and approximately $160 \mathrm{~nm}$ peak to valley amplitude; a) profile section obtained with a 50x Mirau interferometer (center wavelength: $600 \mathrm{~nm}$ ), b) profile section measured with a 50x Linnik interferometer (center wavelength: $395 \mathrm{~nm}$ ); the upper curves represent the height values corresponding to the envelope position, the lower curves are based on phase evaluation techniques.

\section{TRANSFER CHARACTERISTICS OBTAINED FROM SIMULATED SWLI SIGNALS}

In order to clarify the discrepancies between experimental results and theoretical considerations we developed a simulation model, which results in simulated SWLI signals taking the diffraction limited microscopic imaging system of the interferometer into account. ${ }^{14}$ The resulting correlograms can be evaluated in the same way as real measurement signals.

Fig. 16 shows a rectangular amplitude grating with a cycle length of $0.66 \mu \mathrm{m}$ as well as the resulting intensity curve in the image plane of a microscope with a Rayleigh resolution of $0.66 \mu \mathrm{m}$. As is to be expected only the low frequency component of the rectangular is transferred to the image. Low-pass filtering caused by diffraction leads to a dramatic amplitude reduction of the remaining low-frequency component.

Fig. 17 shows a rectangular height profile and the response of a SWLI instrument, obtained by simulation evaluating either the envelope position or the phase of the simulated signals. The result of envelope evaluation looks quite similar to the response of the imaging system according to Fig. 16. However, if the phase of the interference signals is taken into account, apart from batwing deviations the resulting profile is close to the input profile.

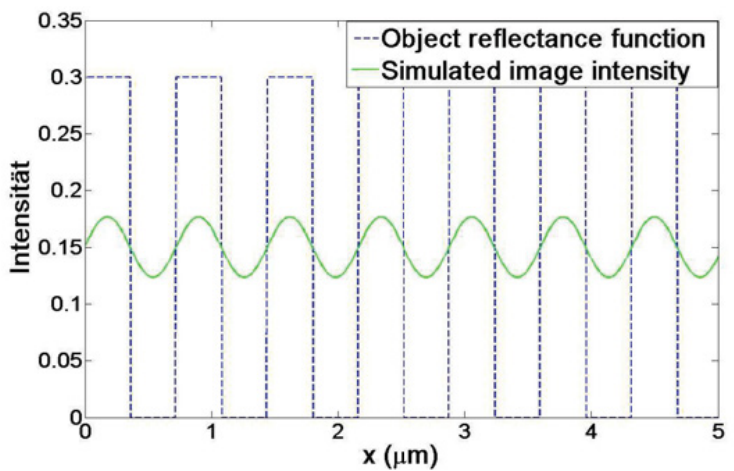

Fig. 16: Simulated object's reflectance function and the corresponding simulated intensity response in the imaging plane assuming that the period of the amplitude grating equals the Rayleigh limit of $0.66 \mu \mathrm{m}$.

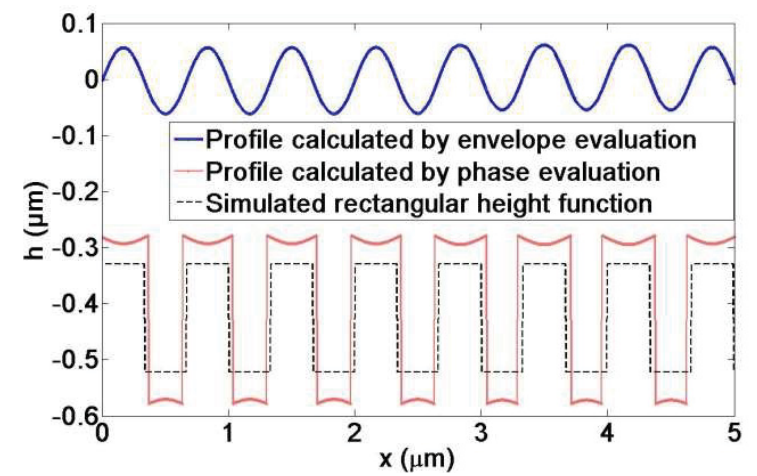

Fig. 17: Simulated rectangular height function (dashed curve) with $0.66 \mu \mathrm{m}$ pitch value, $190 \mathrm{~nm}$ peak-to-valley amplitude and the corresponding response of a white-light interferometer providing a Rayleigh resolution of $0.66 \mu \mathrm{m}$, assuming that the envelope's maximum position is evaluated (upper curve) or the phase of the signals is additionally taken into account (lower curve).

This simulation study confirms the experimental results introduced in section 5. Furthermore, it demonstrates that both, the measuring process based on a depth scan as well as the signal evaluation strategy influence the transfer characteristics of the SWLI measurements.

\section{CONCLUSION}

In this paper we introduce a novel optical area sensor based on SWLI. The sensor is compact enough to be integrated into coordinate measuring machines designed for microstructure measurement. The sensor provides both, a lateral resolution in the submicrometer range and a working distance of more than $5 \mathrm{~mm}$. It could be demonstrated that the sensor resolves rectangular structures of a lateral extension of $300 \mathrm{~nm}$ and a pitch length of $600 \mathrm{~nm}$.

Since these results surpass the theoretical expectations a simulation model was developed, which provides a more realistic impression of transfer characteristics of 
SWLI instruments than current theoretical approaches. It turned out that the transfer characteristics in SWLI strongly is strongly nonlinear. The measuring process based on a depth scan and the signal evaluation (either based on envelope or phase analysis) both influence the measuring results.

\section{ACKNOWLEDGEMENTS}

This work was partly supported by the European commission (project NanoCMM, grant no. FP6026717-2) and partly by the German ministry for economy (BMWi) (project KalWint, grant no. 01FS10045). The support is gratefully acknowledged.

\section{REFERENCES}

[1] L. Deck and P. de Groot, "High-speed noncontact profiler based on scanning white-light interferometry," Appl. Opt. 33, 7334-7338 (1994).

[2] B. S. Lee and T. C. Strand, "Profilometry with a coherence scanning microscope," Appl. Opt. 29, 3784-3788 (1990).

[3] T. Dresel, G. Häusler, and H. Venzke, "Threedimensional sensing of rough surfaces by coherence radar," Appl. Opt. 31, 919-925 (1992).

[4] P. Lehmann and J. Niehues, "Dual-wavelength vertical scanning low-coherence interference microscopy," Proc. SPIE 6616, 6616061-66160610 (2007).

[5] P. de Groot and L. Deck, "Surface profiling by analysis of white-light interferograms in the spatial frequency domain," J. of Mod. Opt. 42, 389-401 (1995).

[6] M. Fleischer, R. Windecker and H. J. Tiziani, "Fast algorithms for data reduction in modern optical three-dimensional profile measurement systems with MMX technology," Appl. Opt. 39, 1290-1297 (2000). [7] P. Lehmann, "Systematic effects in coherence peak and phase evaluation of signals obtained with a vertical scanning white-light Mirau interferometer," Proc. SPIE 6188, 111-1111 (2006).

[8] VDI/VDE guideline 2655 Part 1.1: Optical measurement and microtopographies, "Calibration of interference microscopes and depth measurement standards for roughness measurement" (2008).

[9] P. de Groot, X. C. de Lega, "Interpreting interferometric height measurements using the instrument transfer function," Proc. Fringe 2005, 3037 (2005).

[10] P. Lehmann and J. Niehues, "Limitations and Optimization of Low-coherence Interferometry for High Precision Microscopic Form Measurement," Proc. Fringe 2009, Springer, 244-249 (2009).

[11] P. Lehmann, "Vertical scanning white-light interference microscopy on curved microstructures," Opt. Let. 35, 1768-1770 (2010).

[12] A. Pförtner and J. Schwider, "Dispersion error in white-light Linnik interferometers and its impact for evaluation procedures," Appl. Opt. 40, 6223-6228 (2001).

[13] A. Harasaki and James C. Wyant, "Fringe modulation skewing effect in white-light vertical scanning interferometry," Appl. Opt. 39, 2101-2106 (2000).

[14] W. Xie, P. Lehmann and J. Niehues, „Lateral Resolution and Transfer Characteristics of vertical Scanning White-Light Interferometers, “ Appl. Opt. (submitted).

[15] Datasheet: SiMetrics, "Resolution Standard Type RS-N," (2009). 